\title{
Cross-Layer Optimization Frameworks for Multihop Wireless Networks Using Cooperative Diversity
}

\author{
Long Le, Member, IEEE, and Ekram Hossain, Senior Member, IEEE
}

\begin{abstract}
We propose cross-layer optimization frameworks for multihop wireless networks using cooperative diversity. These frameworks provide solutions to fundamental relaying problems of determining who should be relays for whom and how to perform resource allocation for these relaying schemes jointly with routing and congestion control such that the system performance is optimized. We present a fully distributed algorithm where the joint routing, relay selection, and power allocation problem to minimize network power consumption is solved by using convex optimization. Via dual decomposition, the master optimization problem is decomposed into a routing subproblem in the network layer and a joint relay selection and power allocation subproblem in the physical layer, which can be solved efficiently in a distributed manner. We then extend the framework to incorporate congestion control and develop a framework for optimizing the sum rate utility and power tradeoff for wireless networks using cooperative diversity. The numerical results show the convergence of the proposed algorithms and significant improvement in terms of power consumption and source rates due to cooperative diversity.
\end{abstract}

Index Terms-Cooperative diversity, multihop wireless network, mesh network, convex optimization, cross-layer design, network utility maximization, routing, power control.

\section{INTRODUCTION}

C OOPERATIVE diversity has received significant attention recently as an efficient way to exploit diversity in a wireless network via a virtual distributed antenna array where each antenna belongs to a different node [1][3]. Cooperative diversity was initially intended/proposed for centralized wireless systems. In fact, decode-and-forward and amplify-and-forward cooperative protocols were first proposed in [1], [2] for cellular networks. Outage analysis for these two cooperative protocols was conducted in [3] and coded cooperation protocols were proposed in [4].

Cross-layer design for wireless networks has been another active research area where by exploiting useful interactions of protocols in different layers, the network performance can be improved significantly. In [5], [6], analytical models capturing the interactions between adaptive modulation and

Manuscript received November 20, 2006; revised May 5, 2007 and July 14, 2007; accepted September 8, 2007. The associate editor coordinating the review of this paper and approving it for publication was X. Shen. This work was supported in part by the University of Manitoba Graduate Fellowship (UMGF) and in part by a grant from the Natural Sciences and Engineering Research Council (NSERC) of Canada.

L. Le is with the Department of Electrical and Computer Engineering, University of Waterloo, Waterloo, ON, Canada N2L 3G1 (e-mail: longble@engmail.uwaterloo.ca).

E. Hossain is with the Department of Electrical and Computer Engineering, University of Manitoba, Winnipeg, MB, Canada R3T 5V6 (e-mail: ekram@ee.umanitoba.ca).

Digital Object Identifier 10.1109/TWC.2008.060962. coding in the physical layer and ARQ retransmissions in the link layer under different network conditions were developed for centralized wireless networks. Analysis and optimization of wireless systems using different scheduling policies were conducted in [7], [8]. Cross-layer design and optimization for emerging multihop networks such as wireless mesh networks [9] or multihop cellular networks [10] is much more challenging [11]. In this paper, we tackle optimization problems in multihop wireless networks using cooperative diversity.

Recently, some initial efforts have been made on higher layer protocol design of wireless networks using cooperative diversity. Cooperative medium access control (MAC) protocols were designed in [12], [13]. The key idea behind cooperative MAC problem is to opportunistically exploit relays for transmission if relaying achieves higher throughput than that due to direct transmission. In [11], the authors proposed a MAC protocol for relay selection working with a singlepath routing protocol. This work considered transmission of a single packet to destination nodes. However, these models are difficult to extend for distributed implementation. An optimization framework for cooperative OFDMA-based cellular networks was proposed in [14] where the problem of optimal power allocation, relay and relay strategy selection was jointly tackled. In [15], optimal power allocation solutions for outage probability minimization were obtained for different cooperation strategies. Power allocation schemes for cooperative multihop wireless networks using linear diversity combining techniques and cluster-based routing were investigated in [16] and [17], respectively.

The above works have one or several of the following limitations. First, most of these works were proposed for centralized wireless networks [14], [15]. Second, these works mainly dealt with protocol design issues in one single layer or investigate simple inter-layer interactions, which still lack the system-wide insight [11], [12], [13]. Third, in a multihop context, it was usually assumed that routing solutions are known and also congestion control in the transport layer was not considered [16], [17], [18]. A systematic approach to cross-layer design of multihop wireless networks using cooperative diversity is important to achieve the maximum gain of cooperative diversity in the physical layer and harmonize the interaction with higher layers in such a way that system performance is optimized.

Over the past recent years, nonlinear optimization has been proved to be an important tool for design and analysis of distributed wireless protocols. In fact, dual decomposition technique in convex optimization has been used for 
reverse-engineering of popular protocols such as MAC [19], TCP [20] and for optimal resource allocation and crosslayer design [21], [22]. The work in [24] considered joint congestion control and power control to maximize network utility. In [23], an excellent survey on cross-layer design using convex optimization was provided where several design problems were succinctly presented.

These works, however, did not consider cooperative diversity which can potentially enhance the network performance considerably. In this paper, we apply nonlinear optimization techniques to develop optimization frameworks for multihop wireless networks using cooperative diversity. The first framework is for joint routing and cooperative resource allocation which minimizes the total power consumption. The second framework incorporates congestion control through a utility function to strike a balance between maximizing the sum rate utility and minimizing total power consumption. Inspired by the work in [25], where it was shown that the single "best" relay can achieve the whole diversity-multiplexing tradeoff, we allow only the best relay (if any) to be involved in the relaying process. Note that, in general, cooperation can be among a number of nodes where several nodes (relays) can assist the transmission for each pair of nodes.

The rest of this paper is organized as follows. The system models are presented in Section II. Section III presents the joint routing and cooperative resource allocation framework to minimize network power consumption. The optimization framework for the utility-power tradeoff is presented in Section IV. Section V presents the numerical results. The conclusions are stated in Section VI.

\section{System Model}

Consider a multihop wireless network as a directed graph $G=(V, L)$, where $V$ is the set of nodes and $L$ is the set of directed links. There is a link between a pair of nodes if the communication link can be established between them. We denote the link from node $i$ to node $j$ as $(i, j)$ and the corresponding channel gain by $g^{\prime}(i, j)$. We define $O(i)$ as the set of links going out of node $i$ and $I(i)$ as the set of links going into node $i$. For ease of referencing and understanding of the paper, we abuse the notation a bit by using the same notations for Lagrangian, dual function and others in different problem formulations. However, the notations should be unambiguous from the context they are used.

\section{A. Transmission Rate with Cooperative Diversity}

We assume that transmissions on different links in a common neighborhood use different channels (i.e., in code, frequency or time domain). To forward data from node $i$ to node $j$ on link $(i, j)$, either direct transmission is used or a particular node $k$ helps node $i$ to forward data to node $j$ using decode-and-forward (DF) cooperative diversity. We assume that an available channel pool has been assigned for different links such that simultaneous transmissions are possible where transmission on one link causes very weak interference to others. This assumption on simultaneous transmissions can be achieved, for example, by using different spreading codes with low cross-correlation for different links in a code-division multiple access (CDMA) network. The code allocation algorithms for such a scenario are available in the literature [26]. Another possible scenario justifying this assumption is a multi-channel multi-radio wireless network where orthogonal channels are allocated for simultaneous transmissions on different links in each neighborhood using separate radios in each node [27]. When there is only one or only few channels available, a collision-free transmission schedule should be constructed which is an NP-hard problem even without node cooperation and under a simple one or twohop interference model [31], [32]. Construction of efficient transmission schedule under SINR interference model (i.e., physical interference model [30]) is even more challenging. Due to the complexity of the underlying problem and space constraint, we leave the investigation of cooperative network design under general interference models for future work.

We develop distributed algorithms for optimizing different design objectives as will be mentioned in the next subsection. These algorithms are run when traffic patterns or topology of the network changes. This happens when some network nodes begin or finish transmitting data to their desired destinations or the network topology changes due to node mobility. In essence, the frequent activation of these algorithms offsets the network design parameters (e.g., link flows, power) to compensate for network changes. We assume that total interference and noise power at the receiving end of each link remains static during the running time of the algorithm. This assumption holds when traffic and network topology change slowly [28], [29] and orthogonal channel allocation for simultaneous transmissions in each common neighborhood is performed. In addition, interference and noise power is assumed to be estimated by the receiving nodes and fed back to the transmitting nodes periodically.

Let the transmission power for direct transmission on link $(i, j)$ be $P_{d}(i, j)$, and the total interference and AWGN noise at the receiving side of this link be $N_{0}(i, j)$. Then, the achieved rate $(\mathrm{b} / \mathrm{s} / \mathrm{Hz})$ for direct transmission is

$$
r_{d}(i, j)=\log _{2}\left(1+\frac{g^{\prime}(i, j) P_{d}(i, j)}{\Gamma N_{0}(i, j)}\right)
$$

where $\Gamma$ is the gap to capacity. For notational convenience, we will absorb $\Gamma N_{0}(i, j)$ into $g^{\prime}(i, j)$ and denote the corresponding quantity as $g(i, j)$. Thus, we can write

$$
r_{d}(i, j)=\log _{2}\left(1+g(i, j) P_{d}(i, j)\right) .
$$

For the assumed DF cooperative diversity scheme, time is slotted and node $i$ transmits data packets in the first time slot which are received by node $j$ and relay node $k$. Relay node $k$ decodes the packets and forward them to node $j$ in the second time slot. Let $P_{r,(i, j)}(k, j)$ be the power used by relay node $k$ to forward packets to node $j$ for direct link $(i, j)$. At node $j$, for decoding, the received signal in the second time slot will be combined with that in the first time slot. The achieved data rate at relay node $k$ in the first time slot and at node $j$ in the second time slot after using maximum ratio combining are given, respectively, by

$$
r_{r}(i, k)=\log _{2}\left(1+g(i, k) P_{d}(i, j)\right)
$$




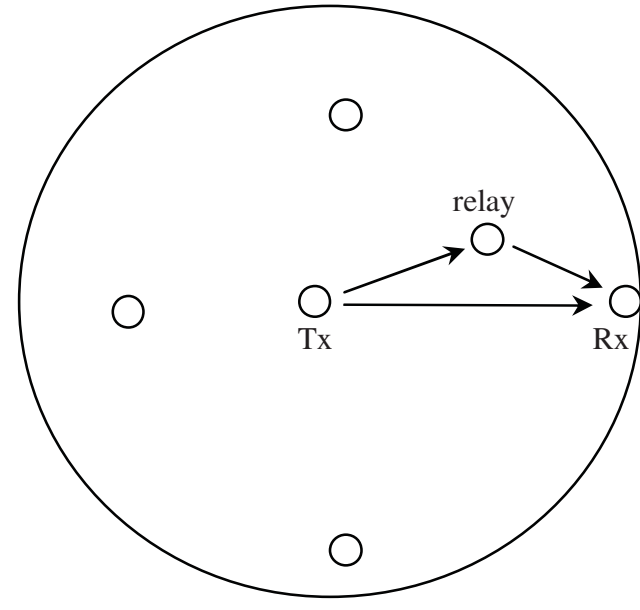

Fig. 1. Cooperative diversity and useful cooperative region.

$$
r_{c}(i, j)=\log _{2}\left(1+g(i, j) P_{d}(i, j)+g(k, j) P_{r,(i, j)}(k, j)\right) .
$$

Note that for relaying to be useful, the achieved rate at relay node $k$ must be higher than that due to direct transmission. This condition can be easily found to be $g(i, j)<g(i, k)$ which will be used to limit the search for the best relay node of each link. To illustrate the useful cooperative region we assume that channel gains are simply due to large-scale path loss. Only nodes inside the circle illustrated in Fig. 1 are useful relays for the considered wireless link. It is also intuitive that wireless nodes in the forward direction toward the receiving node are potentially good relays for the link. Note that the assumption of large-scale path loss in this figure only helps to visualize the useful cooperation region. The cross-layer design frameworks developed later in this paper can be used for any other channel models (e.g., with small-scale fading, shadowing, etc). For energy-efficient communications, we would allocate the right amount of power to relay node $k$ to forward data to destination node $j$ such that

$$
r_{c}(i, j)=r_{r}(i, k) .
$$

Using (3) and (4), this condition is equivalent to

$$
g(i, k) P_{d}(i, j)=g(i, j) P_{d}(i, j)+g(k, j) P_{r,(i, j)}(k, j) .
$$

Let the maximum achievable data rate on link $(i, j)$ be $r(i, j)$. This achievable rate on each link depends on the transmission power, link gain, and the transmission strategy (i.e., direct or cooperative transmission through a relay node). Thus, physical layer design goals considered in this paper are to choose the optimal transmission strategy for each link and to allocate the optimal transmission power level for the chosen strategy such that the system performance captured through appropriate objective functions is optimized. From the above analysis, the achievable transmission rate on link $(i, j)$ for different transmission strategies can be written as

$r(i, j)=\left\{\begin{array}{l}r_{d}(i, j), \quad \text { for direct transmission } \\ \frac{r_{c}(i, j)}{2}=\frac{r_{r}(i, j)}{2}, \quad \text { for cooperative transmission. }\end{array}\right.$

Note that the actual data rate achieved by cooperative diversity is $r_{c}(i, j) / 2$ because two time slots are required to transmit the data. For the same reason, the average total power used for cooperative transmission can be written as

$$
P_{t}(i, j)=\left(P_{d}(i, j)+P_{r,(i, j)}(k, j)\right) / 2 .
$$

Note that transmission power for the case of direct transmission is simply $P_{t}(i, j)=P_{d}(i, j)$. Now, in the following subsection we define the network flow concept and utility function to construct the objective functions.

\section{B. Network Flow and Utility Function}

We use a network flow model for routing data to a single node in a network such as an access point in a wireless LAN or a data sink in a wireless sensor network. In this model, each node $i \neq d$ generates data with an average rate of $S_{i}$ to destination $d$. The total data rate received at destination node $d$ is, therefore, $S_{d}=-\sum_{i \neq d} S_{i}$. We assume that a multi-path routing protocol is used in the network layer where traffic from each source node is split into several flows which follow different multihop paths to reach the desired destination. Define link flow $x(i, j)$ to be the average aggregate traffic rate on link $(i, j)$. The aggregate data transmitted on each link may come from different source nodes under the multipath routing assumption. For flow conservation, the total flow going into a node is the same as total flow going out of that node. Hence,

$$
\sum_{j \in O(i)} x(i, j)-\sum_{j \in I(i)} x(j, i)=S_{i}, \quad i \in V .
$$

The flow on any link $(i, j)$ should be smaller than its transmission rate

$$
x(i, j) \leq r(i, j), \quad(i, j) \in L .
$$

Different objective functions can be optimized depending on the application context. In wireless networks, power minimization is usually one of the biggest concerns because mobile devices (e.g., sensors) are energy-limited and also transmitted energy by a user causes interference to other users. Recall that $P_{t}(i, j)$ is the total consumed power to transmit data on link $(i, j)$. When power consumption is the major concern, the objective function can be $\min \sum_{(i, j) \in L} P_{t}(i, j)$.

Another objective can be to maximize the sum utility of source rates. In this case, the objective function can be written as $\max \sum_{i \in V} U_{i}\left(S_{i}\right)$, where different utility functions $U_{i}\left(S_{i}\right)$ as in [20] for congestion avoidance and fairness control of different data sessions can be used. Another possible objective can be to strike a balance between maximizing sum utility of source rates and minimizing power consumption. For convenience, we will denote the link and node quantities such as link power, link flow, source rate for all links (nodes) in the network into the corresponding vectors. For example, the vector of link flows will be denoted as $\mathbf{x}$ whose element $x(i, j)$ is the link flow for link $(i, j)$.

\section{JoInt Routing AND CoOperative Resource ALLOCATION}

In this section, we present a distributed solution to the joint routing and cooperative resource allocation problem. For this problem, the source rates $S_{i}$ are assumed to be fixed and the optimization problem, in essence, is the joint routing, relay 
selection, and power allocation in a multihop wireless network. The goals are to find optimal transmission strategy for each link (i.e., either direct or cooperative transmission), the optimal power allocation for the chosen strategy, and the link flow to route data generated by source nodes to the corresponding destination node. This is a cross-layer design problem for both physical layer (i.e., relay selection, power allocation) and network layer (i.e., routing of traffic flows). The problem can be stated as follows:

$$
\begin{array}{ll}
\operatorname{minimize} & \sum_{(i, j) \in L} P_{t}(i, j) \\
\text { subject to } & \sum_{j \in O(i)} x(i, j)-\sum_{j \in I(i)} x(j, i)=S_{i}, \quad i \in V \\
& x(i, j) \leq r(i, j), \quad(i, j) \in L \\
& \mathbf{x} \succeq \mathbf{0}, \quad \mathbf{P}_{\min } \preceq \mathbf{P} \preceq \mathbf{P}_{\max }
\end{array}
$$

where $\preceq, \succeq$ denote component-wise inequalities, $\mathbf{x}$ is the vector of link flows, $\mathbf{P}_{\min }\left(\mathbf{P}_{\max }\right)$ denotes the lower (upper) limit for the power vector with element $\mathbf{P}_{\min }(i, j)\left(\mathbf{P}_{\max }(i, j)\right)$ being the minimum (maximum) allowed power to transmit or relay packets on link $(i, j)$. We assume that this problem is feasible. The feasibility is assumed to be maintained by an appropriate admission control algorithm.

We will form the dual problem by introducing the Lagrange multipliers for constraints in (9) and (10). The corresponding Lagrangian can be written as

$$
\begin{aligned}
& L(\mathbf{x}, \mathbf{P}, \boldsymbol{\lambda}, \boldsymbol{\mu}) \\
& =\sum_{(i, j) \in L} P_{t}(i, j)+\sum_{(i, j) \in L} \lambda_{(i, j)}[x(i, j)-r(i, j)] \\
& \quad+\sum_{i \in V} \mu_{i}\left[\sum_{j \in O(i)} x(i, j)-\sum_{j \in I(i)} x(j, i)-S_{i}\right]
\end{aligned}
$$

where $\mathbf{x}$ is the vector of link flows, $\mathbf{P}$ is the vector of allocated powers, $\boldsymbol{\lambda}$ and $\boldsymbol{\mu}$ are the vectors of Lagrange multipliers. The elements of these vectors for each link $(i, j)$ and node $i$ (i.e., $x(i, j), P(i, j), \lambda_{(i, j)}$, and $\left.\mu_{i}\right)$ are maintained at node $i$. From this Lagrangian, we can define the dual function $D(\lambda, \mu)$ as follows:

$$
D(\boldsymbol{\lambda}, \boldsymbol{\mu})=\min _{\{\mathbf{x}, \mathbf{P}\}} L(\mathbf{x}, \mathbf{P}, \boldsymbol{\lambda}, \boldsymbol{\mu}) .
$$

The optimization problem defined in (11) is a convex one where the strong duality holds, and therefore, the duality gap is zero [33]. The original optimization problem in (11) is called a primal problem and its solution can be recovered via what is called a dual problem which can be written as

$$
\begin{array}{ll}
\text { maximize } & D(\boldsymbol{\lambda}, \boldsymbol{\mu}) \\
\text { subject to } & \boldsymbol{\lambda} \succeq 0
\end{array}
$$

where the Lagrange multipliers for the inequality constraints in (10) are constrained to be non-negative. The decision variables for the primal and dual problems are called primal variables ( $\mathrm{x}$ and $\mathbf{P}$ ) and dual variables $(\boldsymbol{\lambda}$ and $\boldsymbol{\mu}$ ), respectively.

Thus, the underlying optimization problem can be solved directly (i.e., by solving the primal problem (11)) or its solution can be obtained through solving the dual problem (14). Solving the primal problem usually results in a centralized algorithm where all network information such as link gains, interference and noise powers at receiving ends of all links should be sent to a particular node to calculate the link flow and allocated power solutions and these solutions should be distributed to the corresponding nodes in the network. The centralized algorithm, therefore, incurs huge communication overhead and lack resilience to network changes. Tackling the dual problem using a dual decomposition method leads to distributed algorithms which are more useful for wireless networks without infrastructure such as ad hoc networks. In these distributed algorithms each node performs iterative exchanges of variables with its immediate neighbors. The dual decomposition method will be used to construct a distributed routing algorithm in the following.

\section{A. Dual Decomposition and Subgradient Method}

Since the objective of the primal problem (11) is not strictly convex, the primal variables (i.e., flow and power vectors $\mathbf{x}$, P) may not be immediately available from the dual problem solutions. To handle this difficulty, we use the approaches in [34] by adding a small regularization term $\epsilon \sum_{(i, j) \in L} x(i, j)^{2}$ into the objective function in (11). By letting $\epsilon \rightarrow 0$, the optimal solution to the regularized problem tends to that in (11). The Lagrangian of the regularized primal problem can be written as

$$
\begin{aligned}
L(\mathbf{x}, \mathbf{P}, \boldsymbol{\lambda}, \boldsymbol{\mu}) & \\
= & \sum_{(i, j) \in L} P_{t}(i, j)+\epsilon \sum_{(i, j) \in L} x(i, j)^{2} \\
& +\sum_{(i, j) \in L} \lambda_{(i, j)}[x(i, j)-r(i, j)] \\
& +\sum_{i \in V} \mu_{i}\left[\sum_{j \in O(i)} x(i, j)-\sum_{j \in I(i)} x(j, i)-S_{i}\right] \\
= & \left\{\epsilon \sum_{(i, j) \in L} x(i, j)^{2}+\sum_{(i, j) \in L} \lambda_{(i, j)} x(i, j)\right. \\
& \left.+\sum_{i \in V} \mu_{i}\left[\sum_{j \in O(i)} x(i, j)-\sum_{j \in I(i)} x(j, i)\right]\right\} \\
& +\left\{\sum_{(i, j) \in L} P_{t}(i, j)-\sum_{(i, j) \in L} \lambda_{(i, j)} r(i, j)\right\} .
\end{aligned}
$$

The first term in the above equation depends only on the primal flow variables $x(i, j)$ and the second term depends only on the primal power variables (because achievable rate $r(i, j)$ for link $(i, j)$ depends on the power vector as modeled in Section II.A). Thus, the dual function can be calculated by decomposing the optimization problem in (13) into following subproblems:

$$
D_{\text {phy }}(\boldsymbol{\lambda})=\min _{\mathbf{P}}\left\{\sum_{(i, j) \in L} P_{t}(i, j)-\sum_{(i, j) \in L} \lambda_{(i, j)} r(i, j)\right\}
$$




$$
\begin{aligned}
D_{\text {net }}(\boldsymbol{\lambda}, \boldsymbol{\mu})= & \min _{\mathbf{x} \succeq \mathbf{0}}\left\{\epsilon \sum_{(i, j) \in L} x(i, j)^{2}+\sum_{(i, j) \in L} \lambda_{(i, j)} x(i, j)\right. \\
& \left.+\sum_{i \in V} \mu_{i}\left[\sum_{j \in O(i)} x(i, j)-\sum_{j \in I(i)} x(j, i)\right]\right\}
\end{aligned}
$$

and the dual function can be written as

$$
D(\boldsymbol{\lambda}, \boldsymbol{\mu})=D_{\text {net }}(\boldsymbol{\lambda}, \boldsymbol{\mu})+D_{\text {phy }}(\boldsymbol{\lambda}) .
$$

Thus, given the dual variables (i.e., $\left.\lambda_{(i, j)}, \mu_{i}\right)$, the dual function can be calculated by solving the routing subproblem in the network layer (i.e., in (17)) and the relay selection and power allocation subproblem in the physical layer (i.e., in (16)). Since the dual function may not be differentiable, we solve the dual problem using the subgradient projection method [35], [36]. Now, we give the definition of sugradient and find the subgradients of the dual function.

Definition: Given a convex function $f: R^{n} \rightarrow R$, vector $d$ is the subgradient of $f$ at $x$ if $f(y) \geq f(x)+d^{T}(y-x)$, $\forall y \in R^{n}$ where $(.)^{T}$ denotes the transposition.

Lemma 1: Subgradient of $-D(\lambda, \mu)$ at $\lambda_{(i, j)}$ and $\mu(i)$ are

$$
\begin{gathered}
f_{i, j}\left(\lambda_{(i, j)}\right)=r^{*}(i, j)-x^{*}(i, j) \quad \text { and } \\
g_{i}\left(\mu_{i}\right)=\sum_{j \in I(i)} x^{*}(j, i)-\sum_{j \in O(i)} x^{*}(i, j)+S_{i}
\end{gathered}
$$

respectively, where $r^{*}(i, j), x^{*}(i, j)$ are optimal solutions in (16), (17).

Proof: Let us find the subgradient for $D_{1}(\boldsymbol{\lambda}, \boldsymbol{\mu})=-D(\boldsymbol{\lambda}, \boldsymbol{\mu})$ at $\lambda_{(i, j)}$. Consider function $D_{1}(.,$.$) at two different values \boldsymbol{\lambda}$ and $\lambda^{\prime}$. We have

$$
D_{1}(\boldsymbol{\lambda}, \boldsymbol{\mu})=-D(\boldsymbol{\lambda}, \boldsymbol{\mu})=\max _{\{\mathbf{x}, \mathbf{P}\}}\{-L(\mathbf{x}, \mathbf{P}, \boldsymbol{\lambda}, \boldsymbol{\mu})\}
$$

$D_{1}\left(\boldsymbol{\lambda}^{\prime}, \boldsymbol{\mu}\right)=-D\left(\boldsymbol{\lambda}^{\prime}, \boldsymbol{\mu}\right)=\max _{\{\mathbf{x}, \mathbf{P}\}}\left\{-L\left(\mathbf{x}, \mathbf{P}, \boldsymbol{\lambda}^{\prime}, \boldsymbol{\mu}\right)\right\}$.

Let the optimal values of $\mathbf{x}, \mathbf{P}$ in (21) and (22) be $\mathbf{x}^{*}, \mathbf{P}^{*}$ and $\mathbf{x}^{*^{\prime}}, \mathbf{P}^{*^{\prime}}$, respectively. We have

$$
\begin{aligned}
D_{1}\left(\boldsymbol{\lambda}^{\prime}, \boldsymbol{\mu}\right)- & D_{1}(\boldsymbol{\lambda}, \boldsymbol{\mu}) \\
= & -L\left(\mathbf{x}^{*^{\prime}}, \mathbf{P}^{*^{\prime}}, \boldsymbol{\lambda}^{\prime}, \boldsymbol{\mu}\right)+L\left(\mathbf{x}^{*}, \mathbf{P}^{*}, \boldsymbol{\lambda}, \boldsymbol{\mu}\right) \\
\geq & -L\left(\mathbf{x}^{*}, \mathbf{P}^{*}, \boldsymbol{\lambda}^{\prime}, \boldsymbol{\mu}\right)+L\left(\mathbf{x}^{*}, \mathbf{P}^{*}, \boldsymbol{\lambda}, \boldsymbol{\mu}\right) \\
= & -\sum_{(i, j) \in L} \lambda_{(i, j)}^{\prime}\left(x^{*}(i, j)-r^{*}(i, j)\right) \\
& +\sum_{(i, j) \in L} \lambda_{(i, j)}\left(x^{*}(i, j)-r^{*}(i, j)\right) \\
= & \sum_{(i, j) \in L}\left(\lambda_{(i, j)}^{\prime}-\lambda_{(i, j)}\right)\left(r^{*}(i, j)-x^{*}(i, j)\right)
\end{aligned}
$$

where $r^{*}(i, j)$ is the achievable rate on link $(i, j)$ with power vector $\mathbf{P}^{*}$. Note that (23) holds because $-L\left(\mathbf{x}^{*^{\prime}}, \mathbf{P}^{*^{\prime}}, \boldsymbol{\lambda}^{\prime}, \boldsymbol{\mu}\right) \geq-L\left(\mathbf{x}^{*}, \mathbf{P}^{*}, \boldsymbol{\lambda}^{\prime}, \boldsymbol{\mu}\right)$ and (24) holds due to the definition of Lagrangian in (15). From the definition of subgradient, the subgradient of $D_{1}(\boldsymbol{\lambda}, \boldsymbol{\mu})=-D(\boldsymbol{\lambda}, \boldsymbol{\mu})$ at $\lambda_{(i, j)}$ is $f_{i, j}\left(\lambda_{(i, j)}\right)=r(i, j)-x(i, j)$. The subgradient for $D_{1}(\boldsymbol{\lambda}, \boldsymbol{\mu})$ at $\mu(i)$ can be obtained in a similar way.

The subgradient projection method is similar to the gradient projection method but the subgradient instead of the gradient of the objective function is used in each iteration. Given the locally optimal solutions $\mathbf{x}^{*}(t)$ and $\mathbf{P}^{*}(t)$ of the networking and the physical layer subproblems, the subgradient projection algorithm updates $\lambda_{(i, j)}$ and $\mu(i)$ as follows:

$$
\begin{gathered}
\lambda_{(i, j)}(t+1)=\left[\lambda_{(i, j)}(t)-\beta(t) f_{i, j}\left(\lambda_{(i, j)}\right)\right]^{+} \\
\mu_{i}(t+1)=\mu_{i}(t)-\beta(t) g_{i}\left(\mu_{i}(t)\right)
\end{gathered}
$$

where $[x]^{+}=\max (0, x)$ and $\beta(t)$ is the appropriate step-size in iteration $t$.

In fact, the proposed algorithm iteratively updates the dual variables (i.e., using (26), (27)) and primal variables (i.e., by solving (16) and (17) ) until globally optimal solutions are obtained. Specifically, the subgradients of the dual function (i.e., $f_{i, j}\left(\lambda_{(i, j)}\right)$ and $\left.g_{i}\left(\mu_{i}\right)\right)$ reflect the degree by which the constraints in (9), (10) are violated. Updating the dual variables based on the subgradient algorithm also has an interesting economic interpretation where the dual variables represent the shadow prices which strike a balance between the supply (transmission power) and demand (link flow) in such a way that globally optimal solutions can be achieved. We will solve these two subproblems in the next subsection. We will refer to $\lambda_{(i, j)}$ as the link price and $\mu_{i}$ as the node price in the sequel.

\section{B. Networking and Physical Subproblem Solutions}

We will now solve the netwoking (i.e., routing) subproblem in (17) and the physical layer subproblem in (16). The routing subproblem in (17) can be rewritten as follows:

$$
D_{\text {net }}(\boldsymbol{\lambda}, \boldsymbol{\mu})=\sum_{(i, j) \in L} D_{\text {net }}(i, j)
$$

where

$D_{\text {net }}(i, j)=\min _{x(i, j) \geq 0}\left\{\epsilon x(i, j)^{2}+\lambda_{(i, j)} x(i, j)+\mu_{i} x(i, j)\right.$

$$
\left.-\mu_{j} x(i, j)\right\} \text {. }
$$

The routing subproblem, therefore, can be decomposed into multiple link subproblems. Given the link and node prices $\lambda_{(i, j)}, \mu_{i}, \mu_{j}$ (these variables are maintained at node $i$ ), node $i$ calculates the locally optimal link flow as

$$
x^{*}(i, j)=\left[\frac{\mu_{j}-\mu_{i}-\lambda_{(i, j)}}{2 \epsilon}\right]^{+} .
$$

The physical layer subproblem in (16) is more difficult to solve because it involves both relay selection and power allocation. This problem can be also decomposed into multiple link subproblems as

$$
D_{\text {phy }}(\boldsymbol{\lambda})=\sum_{(i, j) \in L} D_{\text {phy }}(i, j)
$$

where

$$
D_{\text {phy }}(i, j)=\min _{\mathbf{P}}\left\{P_{t}(i, j)-\lambda_{(i, j)} r(i, j)\right\} .
$$


For each link $(i, j)$, either direct transmission from node $i$ to node $j$ or cooperative transmission with the help a particular relay node $k$ can be pursued. Here, given the link price $\lambda_{(i, j)}$ for each link $(i, j)$, we need to find the best transmission strategy and the corresponding allocated power for it. From (32), if direct transmission is pursued for link $(i, j)$, we have

$$
D_{\text {phy }}(i, j)=\min _{P_{d}(i, j)}\left\{P_{d}(i, j)-\lambda_{(i, j)} r(i, j)\right\} \text {. }
$$

Otherwise, if a neighboring node $k$ is involved in the cooperative transmission, we have

$$
\begin{array}{r}
D_{\text {phy }}(i, j)=\min _{P_{d}(i, j), P_{r,(i, j)}(k, j)}\left\{\left(P_{d}(i, j)\right.\right. \\
\left.\left.+P_{r,(i, j)}(k, j)\right) / 2-\lambda_{(i, j)} r(i, j)\right\} .
\end{array}
$$

Given link price $\lambda_{(i, j)}$, the best transmission strategy is the one which results in the smallest $D_{\text {phy }}(i, j)$. Since node $i$ has a finite number of neighbors which can serve as a relay for cooperative transmission on link $(i, j)$, the best transmission strategy can be easily searched for. Note that as discussed in Section II.A, a possible relay node $k$ must satisfy $g(i, k)>g(i, j)$ for relaying to be useful. This condition limits the number of potential relay candidates. Now, we show how to calculate the locally optimal allocated power for direct transmission and for cooperative transmission. Based on these possible transmission strategies, the best transmission strategy can be easily found. For direct transmission, we have $r(i, j)=r_{d}(i, j)=\log _{2}\left(1+g(i, j) P_{d}(i, j)\right)$. Therefore, the optimal power can be easily found by setting the derivative of the objective function in (33) to zero as

$$
P_{d}^{*}(i, j)=\left[\frac{\lambda_{(i, j)}}{\ln 2}-\frac{1}{g(i, j)}\right]_{P_{\min }(i, j)}^{P_{\max }(i, j)}
$$

where $[x]_{a}^{b}$ denotes the projection of $x$ on $[a, b]$. For cooperative transmission through relay node $k$, we have $r(i, j)=$ $r_{r}(i, k) / 2=1 / 2 \log _{2}\left(1+g(i, k) P_{d}(i, j)\right)$. By setting the derivative of the objective function in (34) to zero and using condition (6), the locally optimal allocated powers can be obtained as

$$
\begin{aligned}
& P_{d}^{*}(i, j)=\left[\frac{\lambda_{(i, j)} g(k, j)}{\ln 2(g(i, k)+g(k, j)-g(i, j))}\right. \\
& \left.-\frac{1}{g(i, k)}\right]_{P_{\min }(i, j)}^{P_{\max }(i, j)} \\
& P_{r,(i, j)}^{*}(k, j)=\left[\frac{g(i, k)-g(i, j)}{g(k, j)} P_{d}^{*}(i, j)\right]_{P_{\min }(k, j)}^{P_{\max }(k, j)} .
\end{aligned}
$$

We now summarize the solution for the physical layer subproblem. The optimal allocated power for direct transmission is given in (35). For cooperative transmission through relay node $k$, the optimal allocated powers for transmitter node $i$ and for relay node $k$ are given in (36) and (37), respectively. Given the optimal power solutions for these possible transmission strategies on link $(i, j)$, the corresponding $D_{\text {phy }}(i, j)$ can be calculated by using (33) for direct transmission and (34) for cooperative transmission. Then, the transmission strategy achieving the smallest $D_{\text {phy }}(i, j)$ is chosen and the corresponding transmission rate $r(i, j)$ is used to calculate subgradient $f_{i, j}$ in the subgradient algorithm. The joint routing and cooperative resource allocation algorithm is summarized in Algorithm 1. The convergence of the algorithm is summarized in Property 1 the proof of which can be found, for example, in [35].

Note that we have assumed multiple simultaneous transmissions can occur on each link by using different spreading codes (i.e., transmission of the underlying link and cooperative transmissions assisting other neighboring links). If simultaneous transmissions on each link are not allowed, then a busy node can simply be removed from the list of potential relays of its neighboring links. In the worst case, if a link could not find any neighbor to serve as a relay, it simply adopts direct transmission as the "best" strategy.

Property 1: Given that the sequence for the step-size $\beta(t)$ is chosen to be nonsummable diminishing which satisfies

$$
\lim _{t \rightarrow \infty} \beta(t)=0, \quad \sum_{t=1}^{\infty} \beta(t)=\infty
$$

Algorithm 1 converges to the globally optimal solution.

\section{Algorithm 1: Joint Routing and Cooperative Resource Allocation}

1) Each node $i$ initializes its node price $\mu_{i}(0)$ and link price $\lambda_{(i, j)}(0)$, link flow $x(i, j)(0)$, transmission power $P_{d}(i, j)(0)$ for outgoing link $(i, j)$.

2) Given $\lambda_{(i, j)}(t)$ and $\mu_{i}(t)$, each node $i$ solves the networking and physical subproblems for its outgoing link $(i, j)$ to obtain the locally optimal link flow $x^{*}(i, j)(t)$, transmission strategy, allocated powers for itself $P_{d}^{*}(i, j)(t)$ and for its relay partner $P_{r,(i, j)}^{*}(k, j)(t)$ if cooperative transmission through node $k$ is the best strategy. Node $i$ then transmits the link flow value $x^{*}(i, j)(t)$ to node $j$ and relay power $P_{r,(i, j)}^{*}(k, j)(t)$ to its relay partner $k$.

3) Given the locally optimal link flow, transmission strategy, and allocated powers, each node $i$ updates the link and node prices $\lambda_{(i, j)}(t+1), \mu_{i}(t+1)$ using (26), (27). Node $i$ transmits $\mu_{i}(t+1)$ to all neighboring nodes $j$.

4) Return to 2) until the algorithm converges.

We observe that Algorithm 1 is fully distributed because each node $i$ in the network performs all its calculations using only variables which are available from its immediate neighbors. Specifically, node $i$ finds the optimal transmission strategy and optimal transmission powers using (35) (for direct transmission strategy) and (36), (37) (for cooperative transmission through node $k$ ). These calculations require $g^{\prime}(i, j), g^{\prime}(i, k), g^{\prime}(k, j)$ and the corresponding interference and noise powers (recall that we have absorbed these values into the corresponding channel gains) which can be made available at node $i$ through some estimation technique and local message exchanges. Similarly, calculations of link flow $x_{(i, j)}(t)$ require variables which are available through message exchange operations in steps 2, 3 of the proposed algorithm.

Note that, a similar amount of computational complexity is involved when no user cooperation is employed if distributed implementation is adopted. The only difference between these two cases is that without user cooperation, each node always chooses direct transmission strategy while with user 
cooperation, each node chooses the best transmission strategy (i.e., can be direct or cooperative transmission via a another relay) for outgoing links in each iteration. The amount of message passing is similar for both cases. The nodes would test the convergence by calculating the difference of node/link prices in two consecutive iterations. The algorithm should be terminated if this difference becomes smaller than a predefined value (e.g., $10^{-6}$ ).

As will be seen from numerical results in Section V, the proposed algorithm takes some time to converge under arbitrary initial conditions. Therefore, the proposed algorithm can be used only in slowly mobile or stationary wireless networks (e.g., stationary wireless mesh networks) where partner selection and algorithm activation from arbitrary initial conditions are not frequently performed. Note that if cooperative partners have been selected for all links, the algorithm can quickly converge to its new optimal solution to track small changes in topology and/or channel gains in successive algorithm runs.

\section{Utility-Power TRAdeOFF With COOPERATIVE RESOURCE ALLOCATION}

In this section, we extend the presented algorithm to incorporate congestion control. In essence, the objective function under consideration strikes a balance between maximizing the sum rate utility and minimizing total power consumption. Note that source rates $S_{i}$ are variables here. The utility function $U_{i}\left(S_{i}\right)$ is assumed to be continuously differentiable, increasing, and strictly concave. In order to recover optimal solution for the primal variables, we add a regularization term into the objective function as before. The optimization problem is given as

$$
\begin{array}{cl}
\operatorname{maximize} & \gamma_{1} \sum_{i \in V} U_{i}\left(S_{i}\right)-\gamma_{2} \sum_{(i, j) \in L} P_{t}(i, j) \\
\text { subject to } & \sum_{j \in O(i)} x(i, j)-\sum_{j \in I(i)} x(j, i)=S_{(i, j) \in L} x(i, j)^{2} \\
& x(i, j) \leq r(i, j), \quad(i, j) \in L \\
& \mathbf{x} \succeq \mathbf{0}, \quad \mathbf{S} \succeq \mathbf{0}, \quad \mathbf{P}_{\min } \preceq \mathbf{P} \preceq \mathbf{P}_{\max }
\end{array}
$$

where $\mathbf{S}$ is the vector of source rates with elements $S_{i}$ being the average data rate generated by node $i$ and $\gamma_{1}, \gamma_{2}$ are the parameters controlling the tradeoff. Note that, only the ratio of $\gamma_{1}$ and $\gamma_{2}$ (i.e., $\gamma_{1} / \gamma_{2}$ ) determines the tradeoff because the objective function is the weighted sum of sum rate utility and network transmission power. In particular, for a fixed value of $\gamma_{2}$ (e.g., $\gamma_{2}=1$ ), a larger value of $\gamma_{1}$ results in larger sum rate utility (and hence transmission rate) as well as transmission power in the network.

\section{A. Dual Decomposition and Subgradient Method}

Proceeding in the same line as in the previous section, we form the following Lagrangian

$L(\mathbf{x}, \mathbf{P}, \mathbf{S}, \boldsymbol{\lambda}, \boldsymbol{\mu})$

$$
\begin{aligned}
= & \gamma_{1} \sum_{i \in V} U\left(S_{i}\right)-\gamma_{2} \sum_{(i, j) \in L} P_{t}(i, j) \\
- & \epsilon \sum_{(i, j) \in L} x(i, j)^{2}-\sum_{(i, j) \in L} \lambda_{(i, j)}[x(i, j)-r(i, j)] \\
- & \sum_{i \in V} \mu_{i}\left[\sum_{j \in I(i)} x(j, i)-\sum_{j \in O(i)} x(i, j)+S_{i}\right] \\
= & \left\{\sum_{i \in V} \gamma_{1} U_{i}\left(S_{i}\right)-\mu_{i} S_{i}\right\} \\
& +\left\{\sum_{(i, j) \in L} \lambda_{(i, j)} r(i, j)-\gamma_{2} \sum_{(i, j) \in L} P_{t}(i, j)\right\} \\
& +\left\{-\epsilon \sum_{(i, j) \in L} x(i, j)^{2}-\sum_{(i, j) \in L} \lambda_{(i, j)} x(i, j)\right. \\
& \left.+\sum_{i \in V} \mu_{i}\left[\sum_{j \in O(i)} x(i, j)-\sum_{j \in I(i)} x(j, i)\right]\right\}
\end{aligned}
$$

where besides the vector of link flows $\mathbf{x}$ and the allocated power vector $\mathbf{P}$, the source rate vector $\mathbf{S}$ is introduced into the Lagrangian. The dual function is given by

$$
D(\boldsymbol{\lambda}, \boldsymbol{\mu})=\max _{\{\mathbf{x}, \mathbf{P}, \mathbf{S}\}} L(\mathbf{x}, \mathbf{P}, \mathbf{S}, \boldsymbol{\lambda}, \boldsymbol{\mu}) .
$$

This dual function can be calculated by decomposing the optimization problem in (41) into the following subproblems:

$$
\begin{aligned}
D_{\text {con }}(\boldsymbol{\mu}) & =\max _{\mathbf{S} \succeq \mathbf{0}}\left\{\sum_{i \in V} \gamma_{1} U_{i}\left(S_{i}\right)-\mu_{i} S_{i}\right\} \\
& =\sum_{i \in V} \max _{S_{i} \geq 0}\left\{\gamma_{1} U_{i}\left(S_{i}\right)-\mu_{i} S_{i}\right\}
\end{aligned}
$$

$$
\begin{array}{r}
D_{\mathrm{net}}(\boldsymbol{\lambda}, \boldsymbol{\mu})=\max _{\mathbf{x} \geq \mathbf{0}}\left\{-\epsilon \sum_{(i, j) \in L} x(i, j)^{2}-\sum_{(i, j) \in L} \lambda_{(i, j)} x(i, j)\right. \\
\left.+\sum_{i \in V} \mu_{i}\left[\sum_{j \in O(i)} x(i, j)-\sum_{j \in I(i)} x(j, i)\right]\right\} \\
=\sum_{(i, j) \in L} \max _{x_{(i, j)} \geq 0}\left\{-\epsilon x(i, j)^{2}-\lambda_{(i, j)} x(i, j)\right. \\
\left.+\mu_{i} x(i, j)-\mu_{j} x(i, j)\right\}
\end{array}
$$

$$
\begin{aligned}
D_{\text {phy }}(\boldsymbol{\lambda}) & =\max _{\mathbf{P}}\left\{\sum_{(i, j) \in L} \lambda_{(i, j)} r(i, j)-\gamma_{2} \sum_{(i, j) \in L} P_{t}(i, j)\right\} \\
& =\sum_{(i, j) \in L} \max _{\mathbf{P} \succeq \mathbf{0}}\left\{\lambda_{(i, j)} r(i, j)-\gamma_{2} P_{t}(i, j)\right\}
\end{aligned}
$$

and the dual function can be rewritten as $D(\boldsymbol{\lambda}, \boldsymbol{\mu})=$ $D_{\text {con }}(\boldsymbol{\mu})+D_{\text {net }}(\boldsymbol{\lambda}, \boldsymbol{\mu})+D_{\text {phy }}(\boldsymbol{\lambda})$. Again, the optimization problem defined in (39) is a convex one where the strong duality holds, and therefore, the duality gap is zero. Thus, solutions of the primal problem in (39) can be recovered via 
its dual problem which can be written as $\min _{\boldsymbol{\lambda} \succeq \mathbf{0}} D(\boldsymbol{\lambda}, \boldsymbol{\mu})$. We solve the dual problem using the subgradient projection method. The subgradients of the dual function at $\lambda_{(i, j)}$ and $\mu_{i}$ are given in the following Lemma.

Lemma 2: The subgradient of $D(\lambda, \mu)$ at $\lambda_{(i, j)}$ and $\mu_{i}$ can be shown to be

$$
\begin{gathered}
\phi_{i, j}\left(\lambda_{(i, j)}\right)=r^{*}(i, j)-x^{*}(i, j) \\
\psi_{i}\left(\mu_{i}\right)=\sum_{j \in O(i)} x^{*}(i, j)-\sum_{j \in I(i)} x^{*}(j, i)-S_{i}^{*}
\end{gathered}
$$

where $S_{i}^{*}, x^{*}(i, j), r^{*}(i, j)$ are optimal solutions in (42), (43), (44).

Proof: The proof is similar to that for Lemma 1.

Given the locally optimal solutions $\mathbf{S}^{*}(t), \mathbf{x}^{*}(t)$, and $\mathbf{P}^{*}(t)$ for the congestion control, networking and physical layer subproblems, the algorithm updates $\lambda_{(i, j)}$ and $\mu(i)$ as follows:

$$
\begin{gathered}
\lambda_{(i, j)}(t+1)=\left[\lambda_{(i, j)}(t)-\beta(t) \phi_{i, j}\left(\lambda_{(i, j)}\right)\right]^{+} \\
\mu_{i}(t+1)=\mu_{i}(t)-\beta(t) \psi_{i}\left(\mu_{i}\right)
\end{gathered}
$$

where $\beta(t)$ is the appropriate step-size in iteration $t$.

Similar to the algorithm presented in Section III, the algorithm for the optimization problem in (39) iteratively updates the dual variables (i.e., using (47), (48)) and solves the congestion control subproblem in (42), networking subproblem in (43), and physical layer subproblem in (44) until the globally optimal solutions are achieved. We present how to solve these subproblems in the following subsection.

\section{B. Solutions of Congestion Control, Networking, and Physical Subproblems}

The congestion control subproblem in (42) and networking subproblem in (43) can be decomposed into multiple node and link subproblems, respectively. Given the node and link prices $\mu_{i}, \lambda_{(i, j)}$, the optimal solutions for these subproblems can be written, respectively, as

$$
\begin{gathered}
S_{i}^{*}=\left[U_{i}^{\prime}-1\left(\frac{\mu_{i}}{\gamma_{1}}\right)\right]^{+} \\
x^{*}(i, j)=\left[\frac{\mu_{i}-\mu_{j}-\lambda_{(i, j)}}{2 \epsilon}\right]^{+}
\end{gathered}
$$

where $U_{i}^{\prime}-1$ is the inverse function of derivative of utility function $U_{i}$.

As in the previous section, the physical layer subproblem involves both relay selection and power allocation. The optimization problem in (44) can be decomposed into multiple link subproblems, where each link searches for the best relay and the corresponding allocated power. The optimal amount of power allocated, if direct transmission is pursued, can be written as

$$
P_{d}^{*}(i, j)=\left[\frac{\lambda_{(i, j)}}{\gamma_{2} \ln 2}-\frac{1}{g(i, j)}\right]_{P_{\min }(i, j)}^{P_{\max }(i, j)} .
$$

For cooperative transmission through relay node $k$, the optimal allocated powers can be written as

$$
\begin{gathered}
P_{d}^{*}(i, j) \\
=\left[\frac{\lambda_{(i, j)} g(k, j)}{\gamma_{2}(g(i, k)+g(k, j)-g(i, j)) \ln 2}-\frac{1}{g(i, k)}\right]_{P_{\min }(i, j)}^{P_{\max }(i, j)} \\
P_{r,(i, j)}^{*}(k, j)=\left[\frac{g(i, k)-g(i, j)}{g(k, j)} P_{d}^{*}(i, j)\right]_{P_{\min }(k, j)}^{P_{\max }(k, j)}
\end{gathered}
$$

Given the link price $\lambda_{(i, j)}$, channel gains, and interference plus noise powers, the strategy resulting in the largest

$$
D_{\text {phy }}(i, j)=\max _{\mathbf{P} \succeq \mathbf{0}}\left\{\lambda_{(i, j)} r^{*}(i, j)-\gamma_{2} \sum_{(i, j) \in L} P_{t}^{*}(i, j)\right\}
$$

will be chosen as the best strategy and the optimal solutions for this strategy are used to update the subgradients in (45) and (46). The joint congestion control, routing, and cooperative resource allocation algorithm to solve (39) is summarized in Algorithm 2. As before, the proof of convergence of this algorithm under the nonsummable diminishing step-size condition can be found in [35].

\section{Algorithm 2: Joint Congestion Control, Routing and Co- operative Resource Allocation for Utility-Power Tradeoff}

1) Each node $i$ initializes its node price $\mu_{i}(0)$ and link price $\lambda_{(i, j)}(0)$, source rate $S_{i}(0)$, link flow $x(i, j)(0)$, transmission power $P_{d}(i, j)(0)$ for each outgoing link $(i, j)$.

2) Given $\lambda_{(i, j)}(t)$ and $\mu_{i}(t)$, each node $i$ solves the congestion control subproblem to obtain the locally optimal source rate $S_{i}^{*}(t)$, solves the networking (i.e., routing), and physical subproblems for its outgoing link $(i, j)$ to obtain the locally optimal link flow $x^{*}(i, j)(t)$, transmission strategy, allocated power for itself $P_{d}^{*}(i, j)(t)$ and for its relay partner $P_{r,(i, j)}^{*}(k, j)(t)$ if cooperative transmission through node $k$ is the best strategy. Node $i$ then transmits the link flow $x^{*}(i, j)(t)$ to node $j$ and relay power $P_{r,(i, j)}^{*}(k, j)(t)$ to its relay partner $k$.

3) Given the locally optimal source rate, link flow, transmission strategy, and allocated powers, each node $i$ updates the link and node prices $\lambda_{(i, j)}(t+1), \mu_{i}(t+1)$ as in (47), (48). Node $i$ transmits $\mu_{i}(t+1)$ to all neighboring nodes $j$.

4) Return to 2) until the algorithm converges.

The presented algorithm for the utility-power tradeoff is also fully distributed. Besides routing and power allocation, which are performed by using local information only, the congestion control solution in (49) for node $i$ requires node price $\mu_{i}$ of itself which is immediately available.

\section{Numerical Results}

We consider a wireless network with 25 nodes distributed in an area of $200 \mathrm{~m} \times 200 \mathrm{~m}$. We investigate two topologies, namely, grid topology (Fig. 2) and random topology (Fig. 3). For the random topology, we fix 4 nodes at four corners and one node at the center of the area; the other 20 nodes are positioned randomly with 5 nodes in each area of $100 \mathrm{~m} \times 100 \mathrm{~m}$ as indicated in Fig. 3. There are two source nodes generating 


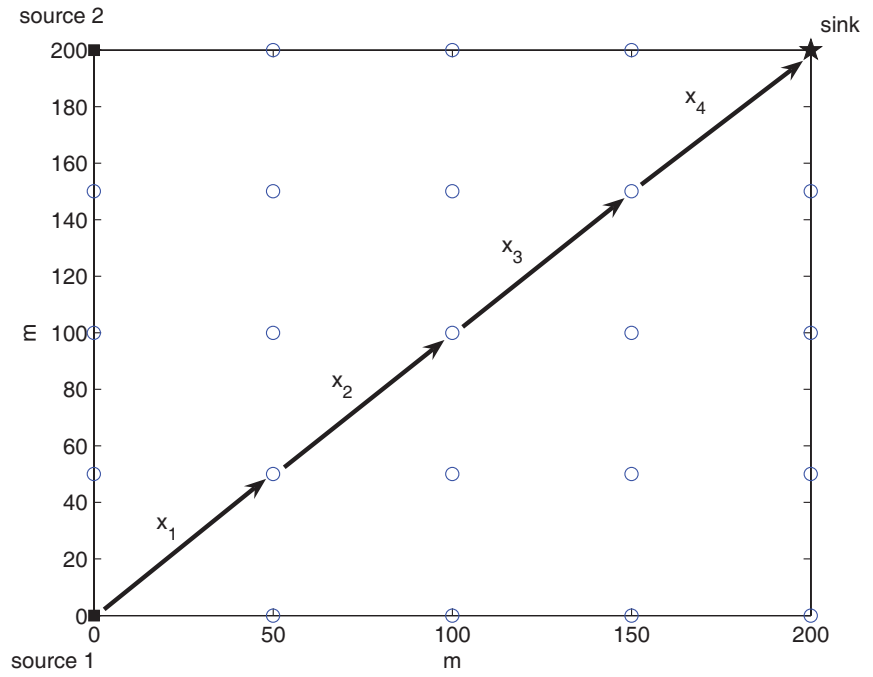

Fig. 2. Grid topology for 25 nodes with a routing path from a source node to the destination node.

data to the single destination node as shown in Figs. 2-3. To show the convergence of the proposed algorithms, we show link flows for a routing path as indicated in Fig. 2. To limit the number of links, we assume that a link exists between a pair of nodes if the distance between them is less than $150 \mathrm{~m}$. The power limits on each link $(i, j)$ are $P_{\min }(i, j)=0 \mathrm{~mW}$, $P_{\max }(i, j)=50 \mathrm{~mW}$ for transmissions from both source nodes and relay nodes.

In order to obtain the numerical results, we run the proposed algorithms with stepsize chosen to be $\beta(t)=$ $\max (0.2 / \sqrt{t}, 0.001)$. The initial value of $\epsilon$ is chosen to be 0.1 . Then it decreases exponentially until it reaches a certain value (e.g., $\epsilon_{0}=0.05$ ) after which it remains the same. There is a tradeoff between the convergence speed and the accuracy of the achieved solution: the higher the $\epsilon_{0}$, the faster the convergence but the less accurate the achieved results are. The channel gain for link $(i, j)$ is modeled as $g(i, j)=K \times d(i, j)^{-3}$, where $K=10^{6}$ and $d(i, j)$ is the distance between node $i$ and $j$ in meter. Note that we have absorbed $\Gamma N_{0}(i, j)$ into these channel gains. The utility function is chosen to be $U_{i}\left(S_{i}\right)=\ln \left(S_{i}\right)$ for the two sources. This utility function provides proportional fairness for the source rates. We compare the network performance with and without cooperative diversity implementation. When cooperative diversity is not employed, direct transmission on each link is always chosen.

The link flows for the routing path indicated in Fig. 2 are shown in Fig. 4. This figure shows the convergence of Algorithm 1. The optimal flows show that data from both sources one and two are actually split into multiple paths to reach the destination node (because the link flows along this routing path are smaller than the source rate which is 4 $\mathrm{b} / \mathrm{s} / \mathrm{Hz}$ ). This implies that a cross-layer framework is necessary to obtain the optimal multipath routing solution. The proposed algorithm actually exploits both cooperative diversity gain from the physical layer and network topology to perform load balancing such that the optimal solution can be achieved.

The total power consumed versus the source rate for both

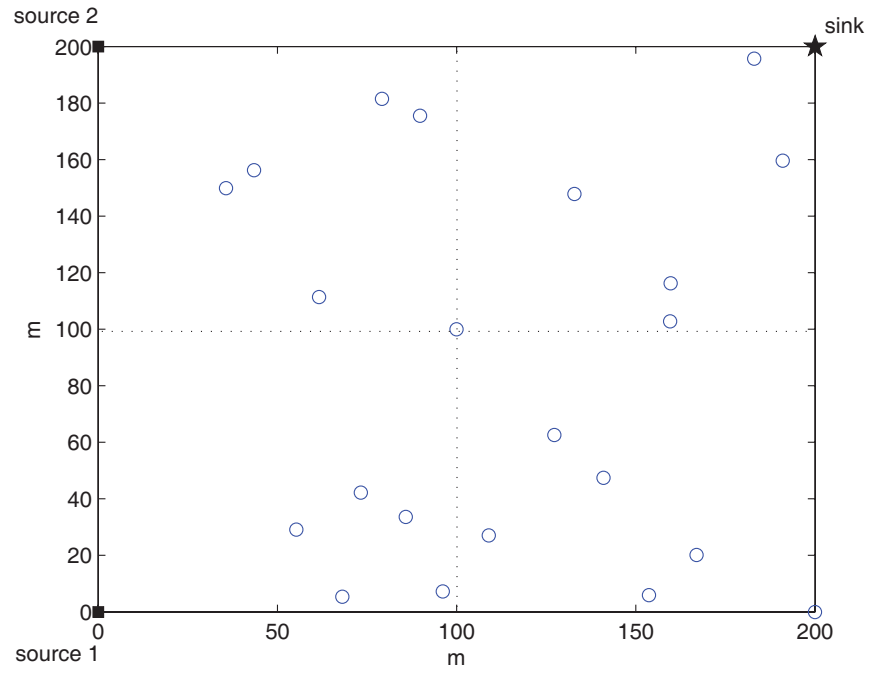

Fig. 3. Random topology for 25 nodes.

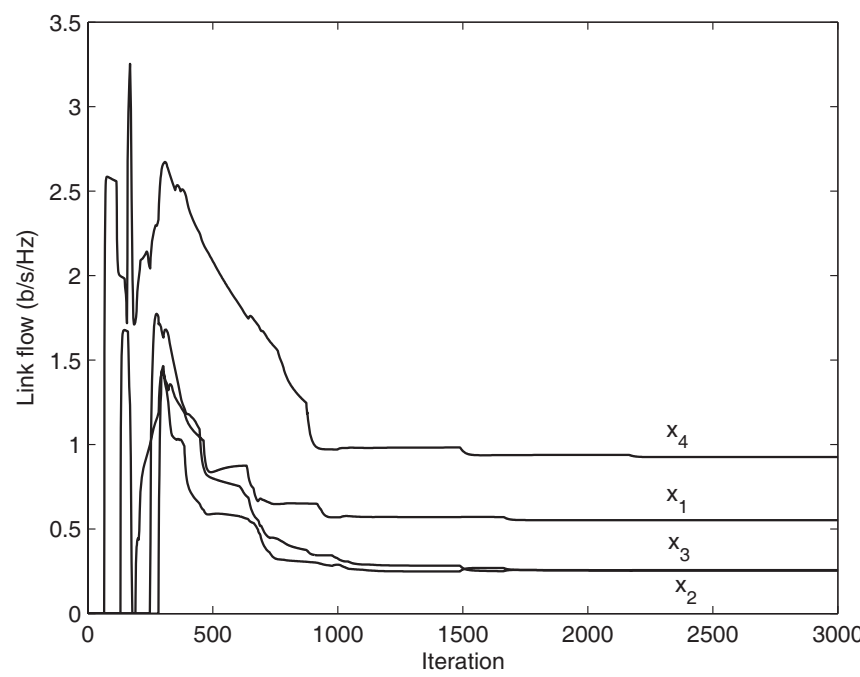

Fig. 4. Link flows for a routing path from source node one to the destination node with cooperative diversity for the grid topology (for $S_{1}=S_{2}=4$ $\mathrm{b} / \mathrm{s} / \mathrm{Hz})$.

the network topologies with and without cooperative diversity implementation is shown in Fig. 5 (denoted by "cooperation" and "non-cooperation", respectively). For the random topology, the total power is obtained by averaging over 20 simulation runs. This figure shows that the random topology actually requires a bit higher power consumption than the grid topology for these particular source-sink pairs. The performance gain resulting from cooperative diversity is very significant which is about $40 \%$ for both the topologies. This performance gain is achieved without sacrificing the distributed nature of the proposed algorithm because only local information is needed to search for the best relay together with the optimal power allocation and routing solution.

To obtain the numerical results for Algorithm 2, we fix $\gamma_{2}=1$ and vary $\gamma_{1}$ to achieve the solutions for different utility-power tradeoff. The link flows for the routing path from source node one to the sink node are shown in Fig. 6. This figure confirms the convergence of Algorithm 2. The total power consumption for utility-power tradeoff optimization is shown 


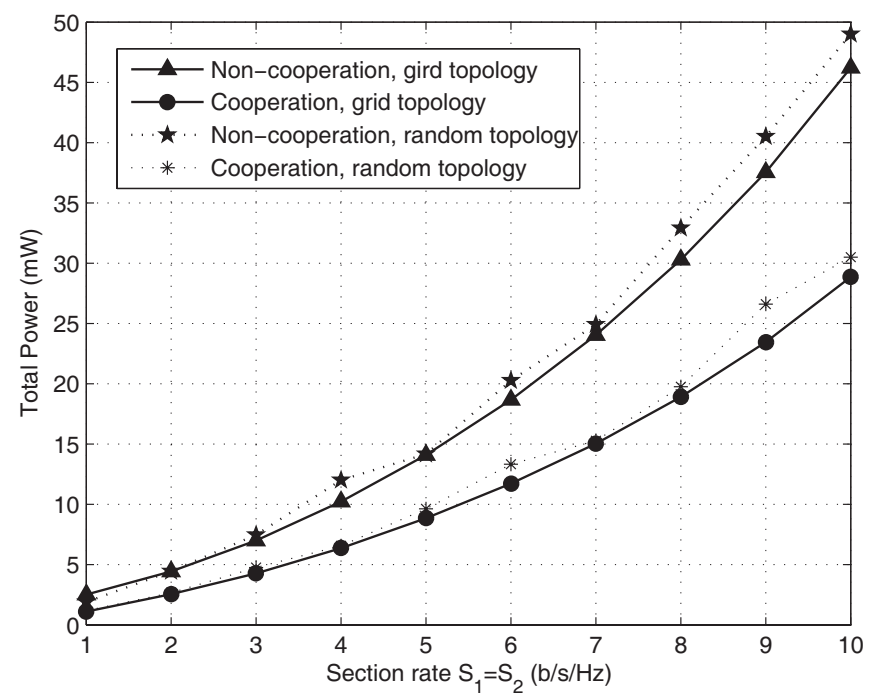

Fig. 5. Total power consumption versus source rate with and without cooperative diversity.

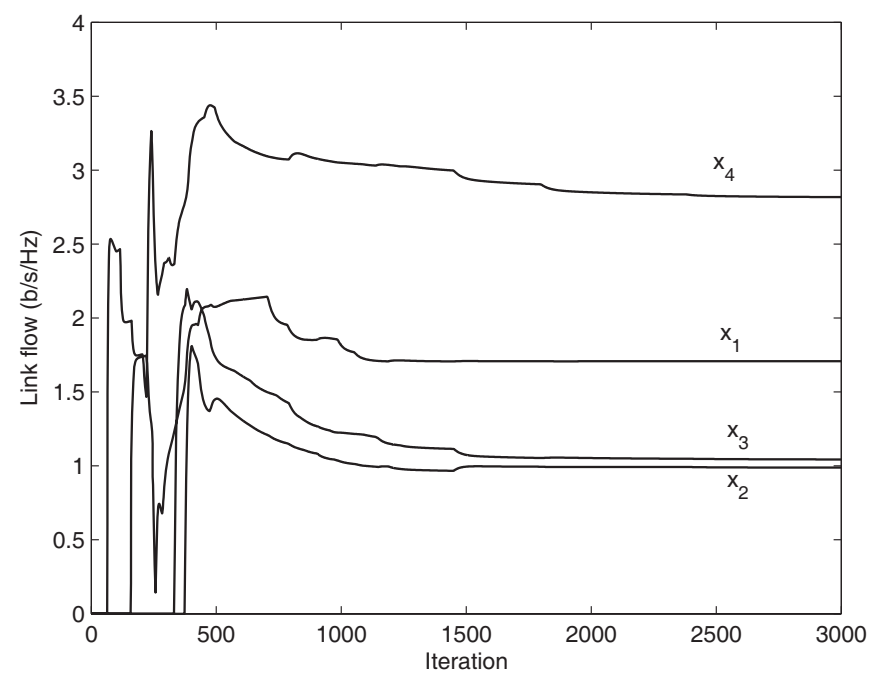

Fig. 6. Link flow with utility and power tradeoff optimization with cooperative diversity for $\gamma_{2}=1$ and $\gamma_{1}=100$.

in Fig. 7 for both topologies with and without cooperative diversity implementation. Again cooperative diversity results in a performance gain although the power gain is smaller compared to that obtained in Fig. 5. This observation can be explained by noticing that the optimization of utility-power tradeoff results in higher total source rate for the cooperation case (Fig. 8). This implies that the performance gain from cooperative diversity is balanced between power and rate gains.

\section{CONCLUSions}

We have developed cross-layer design frameworks for power efficient communications in multihop wireless networks using cooperative diversity. The proposed distributed algorithms converge to the globally optimal solution where the best relay (if any) for each link and the allocated power in the physical layer and the optimal routing solution can be found in a distributed manner. Cross-layer optimization

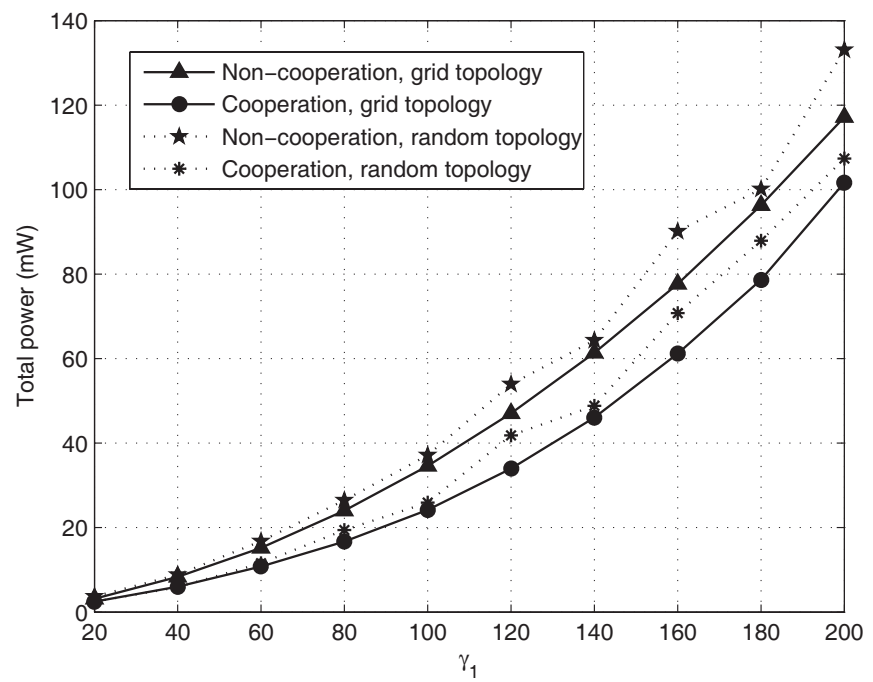

Fig. 7. Total power consumption versus $\gamma_{1}$ for utility-power tradeoff optimization.

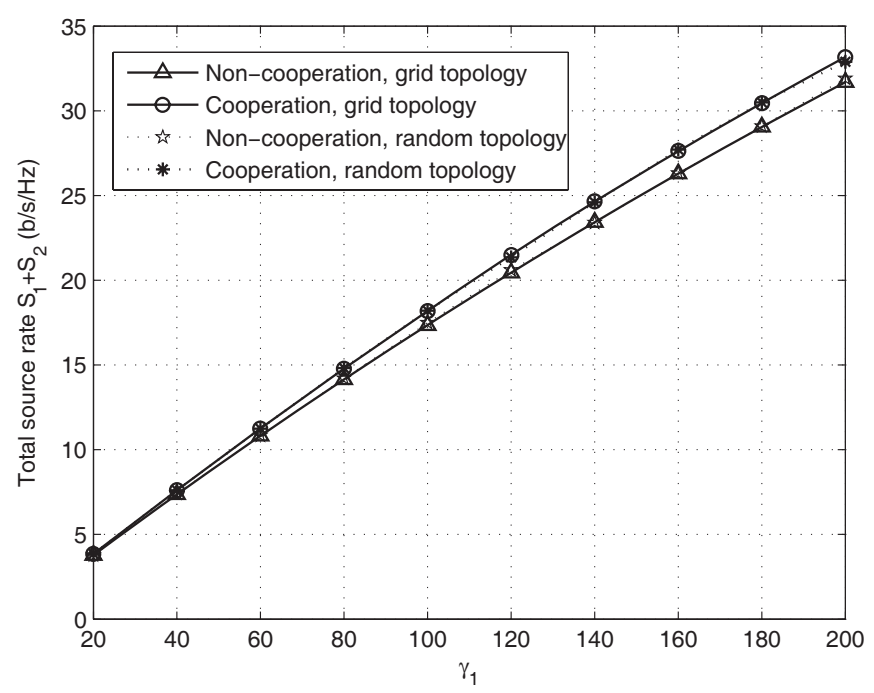

Fig. 8. Total source rate versus $\gamma_{1}$ for utility-power tradeoff optimization.

frameworks have been developed to minimize the total power consumption or to maximize the utility-power tradeoff. For the latter case, the optimal solutions are found to balance performance gain for both power consumption and achieved source rates. The proposed algorithms are very appealing to achieve the cooperative diversity gain, which has been shown to be very significant in this paper.

\section{ACKNOWLEDGMENT}

The authors would like to thank Mung Chiang for his comments and suggestions on different issues addressed in this paper. They also thank the reviewers for their constructive comments which helped to improve the paper.

\section{REFERENCES}

[1] A. Sendonaris, E. Erkip, and B. Aazhang, "User cooperation diversitypart I: system description," IEEE Trans. Commun., vol. 51, no. 11, pp. 1927-1938, Nov. 2003.

[2] A. Sendonaris, E. Erkip, and B. Aazhang, "User cooperation diversitypart II: implementation aspects and performance analysis," IEEE Trans. Commun., vol. 51, no. 11, pp. 1939-1948, Nov. 2003. 
[3] J. N. Laneman, D. N. C. Tse, and G. W. Wornell, " Cooperative diversity in wireless network: efficient protocols and outage behavior," IEEE Trans. Inform. Theory, vol. 50, no. 12, pp. 3062-3080, Dec. 2004.

[4] A. Nosratinia, T. E. Hunter, and A. Hedayat, "Cooperative communication in wireless networks," IEEE Commun. Mag., 2004.

[5] L. B. Le, E. Hossain, and A. S. Alfa, "Radio link level performance evaluation in wireless networks using multi-rate transmission with ARQbased error control," IEEE Trans. Wireless Commun., vol. 5, no. 10, pp. 2647-2653, Oct. 2006.

[6] L. B. Le, E. Hossain, and M. Zorzi, "Queueing analysis for GBN and SR ARQ protocols under dynamic radio link adaptation with non-zero feedback delay," IEEE Trans. Wireless Commun., vol. 6, no. 9, pp. 34183428, Sept. 2007.

[7] L. B. Le, E. Hossain, and A. S. Alfa, "Service differentiation in multi-rate wireless networks with weighted round-robin scheduling and ARQ-based error control," IEEE Trans. Commun., vol. 54, no. 2, pp. 208-215, Feb. 2006.

[8] L. B. Le, E. Hossain, and A. S. Alfa, "Delay statistics and throughput performance for multi-rate wireless networks under multiuser diversity," IEEE Trans. Wireless Commun., vol. 5, no. 11, pp. 3234-3243, Nov. 2006.

[9] I. F. Akyildiz and X. Wang, "A survey on wireless mesh networks" IEEE Commun. Mag., vol. 43, no. 9, pp. 23-30, Sept. 2005.

[10] ] L.Le and E.Hossain, "Multihop cellular networks: Potential gains, research challenges, and a resource allocation framework," IEEE Commun. Mag., vol 45, no. 9, pp. 66-73, Sept. 2007.

[11] G. Jakllari, S. V. Krishnamurthy, M. Faloutsos, P. Krishnamurthy, and O. Ercetin, "A framework for distributed spatio-temporal communications in mobile ad hoc networks," in Proc. IEEE INFOCOM'06.

[12] H. Zhu and G. Cao, "rDCF: a relay-enabled medium access control protocol for wireless ad hoc networks," in Proc. IEEE INFOCOM'05.

[13] P. Liu, Z. Tao, Z. Lin, E. Erkip, and S. Panwar, "Cooperative wireless communications: a cross-layer approach," IEEE Wireless Commun. Mag., vol. 13, no. 4, pp. 84-92, Aug. 2006.

[14] T. C.-Y. Ng and W. Yu, "Joint optimization of relay strategies and resource allocations in a cooperative cellular network," IEEE J. Select. Areas Commun., vol. 25, no. 2, pp. 328-339, Feb. 2007.

[15] R. Annavajjala, P. C. Cosman, and L. B. Milstein, "Statistical channel knowledge-based optimum power allocation for relaying protocols in the high SNR regime," IEEE J. Select. Areas Commun., vol. 25, no. 2, pp. 292-305, Feb. 2007.

[16] S. Savazzi and U. Spagnolini, "Energy aware power allocation strategies for multihop-cooperative transmission schemes," IEEE J. Select. Areas Commun., vol. 25, no. 2, pp. 318-327, Feb. 2007.

[17] A. d. Coso,U. Spagnolini, and C. Ibars "Cooperative distributed MIMO channels in wireless sensor networks," IEEE J. Select. Areas Commun., vol. 25 , no. 2, pp. 402-414, Feb. 2007.

[18] L. Le and E. Hossain, "An analytical model for ARQ cooperative diversity in multihop wireless networks," IEEE Trans. Wireless Commun., vol. 7, no. 5, pp. 1786-1791, May 2008.

[19] X. Wang and K. Kar, "Cross-layer rate control in multi-hop wireless networks with random access," IEEE J. Select. Areas Commun., vol. 24 , no. 8, pp. 1548-1559, Aug. 2006.

[20] J. Mo and J. Walrand, "Fair end-to-end window-based congestion control," IEEE/ACM Trans. Networking, vol. 8, no. 5, pp. 556-567, Oct. 2000.

[21] L. Xiao, M. Johansson, and S. P. Boyd, "Simultaneous routing and resource allocation via dual decomposition," IEEE Trans. Commun., vol. 52, no. 7, pp. 1136-1144, July 2004.

[22] F. P. Kelly, A. Maulloo, and D. Tan, "Rate control for communication networks: shadowing prices, proportional fairness, and stability," J. Oper. Res. Soc., vol. 49, no. 3, pp. 237-252, Mar. 1998.

[23] M. Chiang, S. H. Low, A. R. Calderbank, and J. C. Doyle, "Layering as optimization decomposition: a mathematical theory of network architectures," Proc. IEEE, vol. 95, no. 1, pp. 255-312, Jan. 2007.

[24] M. Chiang, "Balancing transport and physical layers in wireless multihop networks: jointly optimal congestion control and power control," IEEE J. Select. Areas Commun., vol. 23, no. 1, pp. 104-116, Jan. 2005.
[25] A. Bletsas, A. Khisti, D. P. Reed, and A. Lippman, "A simple cooperative diversity method based on network path selection," IEEE J. Select. Areas Commun., vol. 24, no. 3, Mar. 2006.

[26] L. Hu, " Distributed code assignments for CDMA packet radio networks," IEEE/ACM Trans. Networking, vol. 1, pp. 668-677, Dec. 1993.

[27] P. Kyasanur and N. H. Vaidya, "Routing and interface assignment in multi-channel multi-interface wireless networks," in Proc. WCNC'05, Mar. 2005.

[28] D. S. J. D. Couto, D. Aguayo, J. Bicket, and R. Morris, "A highthroughput path metric for multi-hop wireless routing," in Proc. IEEE MOBICOM'O3.

[29] C. E. Koksal, H. Balakrishnan, "Quality-aware routing metrics for timevarying wireless mesh networks," IEEE J. Select. Areas Commun., vol. 24, no. 11, pp. 1984-1994, Nov. 2006.

[30] P. Gupta and P. R. Kumar, "The capacity of wireless networks," IEEE Trans. Inform. Theory, vol 46, no. 2, pp. 388-404, Mar. 2000.

[31] X. Lin and N. B. Shroff, "The impact of imperfect scheduling on cross-layer congestion control in wireless networks," IEEE/ACM Trans. Networking, vol. 14, no. 2, pp. 302-315, Apr. 2006.

[32] A. Gupta, X. Lin, and R. Srikant, "Low-complexity distributed scheduling algorithms for wireless networks," in Proc. IEEE INFOCOM'07.

[33] S. Boyd and L. Vandenberge, Convex Optimization. Cambridge University Press, 2004.

[34] D. P. Bertsekas and J. N. Tsitsiklis, Parallel and Distributed Computation: Numerical Methods. Athena Scientific, 1997.

[35] N. Z. Shor, Minimization Methods for Non-Differentiable Functions. Springer-Verlag, 1985.

[36] D. P. Bertsekas, Nonlinear Programming, 2-nd Edition. Belmont, MA: Athena Scientific, 1999.

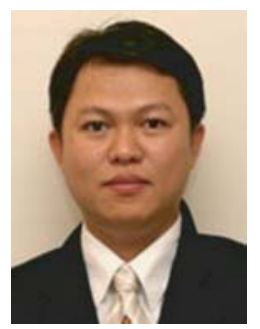

Long Le (S'04-M'07) received the B.Eng. degree with highest distinction from Ho Chi Minh City University of Technology in 1999, the M.Eng. degree from Asian Institute of Technology (AIT) in 2002 and the Ph.D. degree from University of Manitoba in 2007. He is now a postdoc fellow in the Department of Electrical and Computer Engineering at University of Waterloo. He was awarded the university gold medal in the undergraduate program, Keikyu scholarship, University of Manitoba graduate fellowship, Edward R. Toporeck graduate fellowship in engineering, University of Manitoba students' union scholarship, IEEE student travel awards for IEEE WCNC 2003, IEEE ICC 2005. His current research interests include cognitive radio, network coding, link and transport layer protocol issues, cooperative diversity and relay networks, stochastic control and cross-layer design for communication networks. He is a Member of the IEEE.

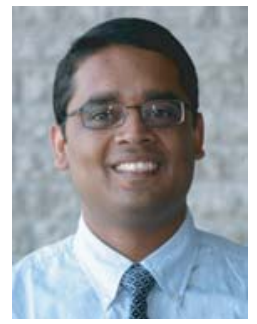

Ekram Hossain (S'98-M'01-SM'06) is currently an Associate Professor in the Department of Electrical and Computer Engineering at the University of Manitoba, Winnipeg, Canada. He received his Ph.D. in Electrical Engineering from the University of Victoria, Canada, in 2000. Dr. Hossain's current research interests include design, analysis, and optimization of wireless communication networks and cognitive radio systems. He is a co-editor/co-author for the books Cognitive Wireless Communication Networks (Springer, 2007, ISBN: 978-0-387-68830-5), Wireless Mesh Networks: Architectures and Protocols (Springer, 2007, ISBN: 9780-387-68839-8), Heterogeneous Wireless Access Networks (Springer, 2008, ISBN: 978-0-387-09776-3), and Introduction to Network Simulator NS2 (Springer, 2008, ISBN: 978-0-387-71759-3). Dr. Hossain serves as an Editor for the IEEE Transactions on Mobile Computing, the IEEE Transactions on Wireless Communications, the IEEE Transactions on Vehicular Technology, IEEE Wireless Communications, and several other international journals. He is a registered Professional Engineer in the province of Manitoba, Canada. 